\title{
Generation of tumor-infiltrating lymphocytes from pancreatic cancer lesions for cellular therapy
}

\author{
Qingda Meng ${ }^{1}$, Elena B Rangelova ${ }^{2}$, Liu zhenjiang ${ }^{1}$, Thomas Poiret ${ }^{1}$, Bartek Jiri ${ }^{1}$, Caroline Verbeke ${ }^{1}$, Ernest Dodoo ${ }^{3}$, \\ Ralf Segersvärd ${ }^{1}$, Markus Maeurer ${ }^{1 *}$
}

From Society for Immunotherapy of Cancer 29th Annual Meeting

National Harbor, MD, USA. 6-9 November 2014

\section{Purpose}

The generation of $\mathrm{T}$ lymphocytes with specific reactivity against autologous tumor is a prerequisite for effective adoptive transfer therapies. Pancreatic cancer-specific lymphocyte cultures from tumor infiltrating lymphocytes (TILs) may represent a viable source of $\mathrm{T}$ cells for the biological therapy for patients with pancreatic cancer.

\section{Methods}

Pancreatic cancer tissue was obtained either by surgery or from biopsy specimens from 16 patients and cultured with cytokines (IL-2, IL-15and IL-21). TIL were expanded using OKT-3 and irradiated allogeneic peripheral blood mononuclear cells (PBMCs). TIL reactivity was gauged for recognition of molecularly defined tumor-associated antigens (TAAs, mesothelin, survivin and NY-ESO-1) by IFNgamma production and intracellular cytokine production (ICS). TCR VB T cell populations were tested by a panel of TCR $\mathrm{Vb}$ specific antibodies, along with $\mathrm{T}$ cell differentiation and exhaustion markers by flow cytometry.

\section{Results}

TIL from $16 / 16$ patients, up to $10 \mathrm{e} 11$ cells, could be successfully expanded using IL-2/15/21. 4 week TIL cultures showed up to $90 \%$ CD $8+$ T cells, yet $1 / 16$ TIL cultures exhibited exclusively CD4+ TIL with a CD45RA-CCR7+ phenotype. 12 / 16 of TILs showed preferential expansion of TCR VB families, i.e. 99.3\% in Vb13.2 in CD8+ TIL, 77\% in VB1, 68.7\% in VB22, 64\% in VB14 for individual patients. Even biopsy specimens (about $10 \mathrm{mg}$ ), yielded at least 1.5 x10e9 CD8 TIL. ICS analysis showed a low frequency (up to 2.5\%) of mesothelin, survivin or NY-ESO-1 reactive CD8+ TIL. TIL from a $1 / 16$ patients showed up to
10\% NY-ESO-1 specific IFNgamma and TNFalpha production in CD4+ and CD8+ T cells. Tumors from these patients are currently sequenced for mutations and subsequent testing for TIL recognition.

\section{Conclusion}

We have optimized methods for the robust and fast generation of TIL from pancreatic cancer lesions, including small biopsy specimens, using a cytokine cocktail of IL-2/ IL-15 and IL-21. TIL showed a Th1-cytokine production pattern and a central memory phenotype. A Phase I clinical safety trial at Karolinska is currently prepared for IL-2/ 15/21-expanded TIL for the cellular therapy for patients with pancreatic cancer.

\section{Authors' details \\ ${ }^{1}$ Karolinska Insitutet, Stockholm, Sweden. ${ }^{2}$ Karolinska Insitutet, Karolinska University Hospital, Stockholm, Sweden. ${ }^{3}$ Dept. of Neurosurgery, Karolinska University Hospital, Sweden.}

Published: 6 November 2014

${ }^{1}$ Karolinska Insitutet, Stockholm, Sweden

Full list of author information is available at the end of the article 